\title{
A formulated red ginseng extract upregulates CHOP and increases TRAIL-mediated cytotoxicity in human hepatocellular carcinoma cells
}

\author{
YUN-SUN LEE ${ }^{1 *}$, DA-GYUM LEE ${ }^{1 *}$, JU-YEON LEE ${ }^{1}$, TAE RYONG KIM ${ }^{2}$, \\ SOON-SUN HONG ${ }^{3}$, SUNG WON KWON ${ }^{2}$ and YOU-SUN KIM ${ }^{1}$ \\ ${ }^{1}$ Institute for Medical Sciences, Ajou University School of Medicine, Suwon 443-749; ${ }^{2}$ College of Pharmacy \\ and Research Institute of Pharmaceutical Sciences, Seoul National University, Seoul 151-742; \\ ${ }^{3}$ Department of Biomedical Sciences, College of Medicine, Inha University, \\ Incheon 400-712, Republic of Korea
}

Received March 18, 2013; Accepted April 29, 2013

DOI: $10.3892 /$ ijo.2013.1964

\begin{abstract}
Tumor necrosis factor-related apoptosis-inducing ligand (TRAIL) is a promising anticancer agent because its cytotoxicity is selective for tumor cells. Despite promising outcomes in clinical trials using this ligand, sustained clinical responses have been impeded because cancer cells acquire resistance to TRAIL-based therapies. Ginseng, a well-known food product consumed globally, has been reported to reduce fatigue and possess antioxidant and antitumor activities. We explored the sensitizing influence of a formulated red ginseng extract (RGE) on TRAIL-derived cell death in hepatocellular carcinoma (HCC) cell lines and the underlying molecular mechanisms responsible for TRAIL sensitization. We found that the RGE promoted TRAIL-derived apoptosis in HepG2, Huh-7 and Hep3B cell lines. We also found that death receptor 5 expression was induced by the RGE and mediated by C/EBP homologous protein (CHOP). shRNA-induced downregulation of CHOP expression effectively suppressed cell death induced by combined treatment with the RGE and TRAIL in the HepG2 cell line, indicating that RGE-related upregulation of the CHOP protein plays an important role in sensitizing TRAIL-derived apoptosis. In summary, we showed that the RGE sensitized human HCC cell lines to TRAIL-derived cell
\end{abstract}

Correspondence to: Professor You-Sun Kim, Institute for Medical Sciences, Ajou University School of Medicine, San 5, Wonchon-dong, Yeongtong-gu, Suwon 443-749, Republic of Korea

E-mail: yousunkim@ajou.ac.kr

Dr Sung Won Kwon, College of Pharmacy, Seoul National University, 599 Gwanangno, Gwanak-gu, Seoul 151-742, Republic of Korea

E-mail: swkwon@snu.ac.kr

*Contributed equally

Key words: ginsenoside, CHOP, apoptosis, DR5 death and could be utilized as a dietary supplement in combination with cancer treatment.

\section{Introduction}

TRAIL, also known as Apo2L and TNFSF10, belongs to the tumor necrosis factor (TNF) superfamily that induces cell death in various types of cancer cell lines in vivo and in vitro but has little or no effect on normal cell lines. The cytotoxic effects of TRAIL are carried out by its functional receptors, death receptor 4 (DR4) and death receptor 5 (DR5) (also known as TRAIL-R1 and TRAIL-R2), and death inducing signaling complex (DISC). DISC formation involves recruitment of an adaptor molecule, Fas-associated death domain (FADD), as well as apical caspase- 8 and caspase-10. DISC assembly results in the auto-catalytic activation of caspase- 8 and caspase-10, which initiates the caspase cascade, leading to apoptosis $(1,2)$. Upon binding to DR4 and DR5, TRAIL activates the nuclear factor (NF)- $\kappa \mathrm{B}$ and JNK signaling pathways (3). NF- $\kappa B$ plays an important role regulating anti- and pro-apoptotic events, depending on the physiological environment (4). NF- $\mathrm{\kappa B}$ mediates the expression of anti-apoptotic genes, such as members of the inhibitor of apoptosis (IAP) family (cIAP-1 and -2 and XIAP), cellular Flice-like inhibitory proteins (c-FLIP) and Bcl-2, all of which determine susceptibility to TRAIL-derived apoptosis $(5,6)$.

TRAIL is regarded as a promising anticancer agent because of its selective cytotoxicity against transformed cell lines; however, the potential clinical use of TRAIL has been blocked because some tumor cell lines become resistant to TRAIL-derived apoptosis $(7,8)$. Resistance is generally induced by low expression of DR4/DR5 or high expression of IAP family members $(8,9)$; thus, the discovery of agents that alleviate TRAIL resistance may allow the use of combined TRAIL-based therapeutic regimens to treat resistant cancers (10). Recombinant human TRAIL and several agonistic monoclonal antibodies are currently in phase II clinical trials. The agonistic antibodies include mapatumumab, which targets DR4, and lexatumumab, apomab, AMG655, CS-1008, and LBY-135, which target DR5 (11). However, increasing evidence indicates 
that death receptor agonists alone may not be sufficient to effectively activate apoptosis in many cancers.

Chemotherapy is not a standard treatment for hepatocellular carcinoma (HCC), and HCC is highly resistant to other conventional anti-neoplastic agents $(12,13)$. Recent studies have shown that many cancer cell lines are not susceptible to the cytotoxic effects of TRAIL (14); thus, the discovery of agents that alleviate TRAIL resistance may be useful for the establishment of TRAIL-based combined regimens for improved HCC treatment.

A formulated red ginseng extract (RGE) derived from heat-processed Panax ginseng C.A. Meyer, is a functional food and dietary supplement consumed worldwide and is used in traditional oriental medicine to increase energy and life expectancy. RGE has been reported to reduce fatigue and to possess antioxidant and antitumor activities (15-17). Ginseng has been reported to possess antioxidant, antistress and immunestimulating activities. These activities require the scavenging of hydroxyl, 1-1-diphenyl-2-picryhydrazyl (DPPH) and superoxide radicals to decrease lipid peroxidation through chelation of transition metal ions and to reduce oxidative DNA damage caused by the Fenton reaction or UV light exposure (18). Ginseng saponins also protect human low-density lipoproteins from oxidative damage in vitro (19) and induce $\mathrm{Cu} / \mathrm{Zn}$-superoxide dismutase expression at the transcriptional level (20).

Findings indicate that the RGE could be useful as a dietary supplement in a combined cancer treatment, as it sensitizes human HCC cell lines to TRAIL-derived cell death via a mechanism involving upregulation of DR5, which then enhances activation of the extrinsic apoptosis pathway. In this case, upregulation of DR5 is induced by C/EBP homologous $(\mathrm{CHOP})$, an endoplasmic reticulum (ER) stress responsive element. shRNA-mediated downregulation of CHOP expression effectively suppresses cell death induced by RGE plus TRAIL in HepG2 cells, indicating that CHOP is essential for RGE-triggered enhancement of TRAIL-derived apoptosis. To summarize, data from the present study suggest that RGE may sensitize cells to TRAIL, thereby diminishing their resistance. RGE could be combined with TRAIL in a cocktail for use as a novel therapeutic strategy to more effectively treat HCC.

\section{Materials and methods}

Reagents. The following reagents were purchased and used according to the manufacturer's instructions: glutathione S-transferase (GST)-TRAIL and anti-DR5 antibodies were from Koma Biotechnologies (Seoul, Korea); anti-caspase 3, anti-PARP and anti-CHOP antibodies were from Cell Signaling Technology; anti-DR4 antibody was from Rockland; anti-tubulin antibody was from Abcam; anti-actin antibody, thapsigargin (Tg), necrostatin-1, NAC and BHA were from Sigma; and zVAD was from R\&D Systems. A formulated RGE was provided by the Korea Ginseng Corporation (Seoul, Korea).

Cell culture. The SK-Hep1, HepG2 and Hep3B cell lines were grown on culture plates in Dulbecco's modified Eagle's medium supplemented with $10 \%$ fetal bovine serum (FBS), 2 mM glutamine, $100 \mathrm{U} / \mathrm{ml}$ penicillin and $100 \mu \mathrm{g} / \mathrm{ml}$ streptomycin. Huh-7 cells were grown on culture plates in RPMI-1640 medium supplemented with $10 \%$ FBS, 2 mM glutamine, $100 \mathrm{U} / \mathrm{ml}$ penicillin and $100 \mu \mathrm{g} / \mathrm{ml}$ streptomycin. A commercially available normal liver cell line (HL-7702) was grown on culture plates in RPMI-1640 medium supplemented with 20\% FBS, 2 mM glutamine, $100 \mathrm{U} / \mathrm{ml}$ penicillin and $100 \mu \mathrm{g} / \mathrm{ml}$ streptomycin.

Western blot analysis. Upon treatment, cell lines were lysed in $20 \mathrm{mM}$ Tris (pH 7.0), $250 \mathrm{mM} \mathrm{NaCl}, 3 \mathrm{mM}$ EDTA, $3 \mathrm{mM}$ EGTA, 2 mM DTT, 0.5\% NP-40, 0.5 mM PMSF, $20 \mathrm{mM}$ $\beta$-glycerol phosphate, $1 \mathrm{mM}$ sodium vanadate and $1 \mu \mathrm{g} / \mathrm{ml}$ leupeptin. Lysates were loaded onto 10 or $12 \%$ SDS-PAGE gels. Following transfer and blotting, the proteins of interest were visualized by enhanced chemiluminescence (ECL, Pierce) and analyzed.

Cytotoxicity assays. Viable cells were measured by the MTT assay (dual labeling method with $2 \mu \mathrm{M}$ calcein-AM and $4 \mu \mathrm{M}$ EthD-1), and MTT absorbance was read at $570 \mathrm{~nm}$. Lactate dehydrogenase (LDH) leakage was quantified using a cytotoxicity detection kit (Promega). Images were recorded by microscope and the data presented are from at least three independent experiments.

Lentiviral shRNA experiments. shRNA-encoding plasmids were purchased from Sigma-Aldrich, and the targets were coding region sequences or the 3 ' untranslated region of CHOP mRNA (NM: 004083). The 293T cell line (LV900A-1) was transfected with lentiviral plasmids using Lipofectamine 2000 transfection reagent (Invitrogen, 11668019). HepG2 cell lines were infected with pseudoviral particles collected from the $293 \mathrm{~T}$ cell line 2 days after transfection in the presence of Polybrene $(8 \mu \mathrm{g} / \mathrm{ml})$. The lentivirus-infected HepG2 cell line was then isolated by puromycin $(1 \mu \mathrm{g} / \mathrm{ml})$ resistance 2 days after infection, and knockdown of CHOP was confirmed by western blot analysis. CHOP knockdown cell lines were seeded on 6-well plates and treated with the RGE or Tg for the times indicated for each experiment and were analyzed by western blot analysis.

\section{Results and Discussion}

Analysis of the formulated $R G E$. A high-performance liquid chromatography/mass spectrometry (LC/MS) method was used for the targeted 14 ginsenosides to determine the ginsenoside content in the formulated RGE. Butanol extracts of white, red and sun ginseng were employed to isolate the standard ginsenosides, and each ginsenoside was isolated and purified by silica gel chromatography or preparative LC $(21,22)$. Calibration curves for each ginsenoside standard were plotted (data not shown), and the ginsenoside contents of the formulated RGE were calculated (Fig. 1, Table I). Korean red ginseng has been reported to induce antitumor and immune-stimulating, antistress and antioxidant activities. The cytoprotective and chemoprotective properties of ginseng have been attributed to its ability to reduce oxidative or nitrosative stress (23-25). Previous studies have suggested that RGE, a major component of ginseng, inhibits cancer cell propagation and metastasis (24). Based on these observations, we tested the cytotoxicity of the RGE in the HepG2 cell lines. As shown in Fig. 2A, RGE concentrations up to $10 \mathrm{mg} / \mathrm{ml}$ showed no significant effects on cells (left panel) and no decrease in viability, as measured by LDH release (right panel). As a positive control, we treated the HepG2 cell lines with TRAIL $(50 \mathrm{ng} / \mathrm{ml})$ and observed the induction of cell 
Table I. LC/MS conditions for the quantification of ginsenoside in RGE.

Instrument

Column

Mobile phase condition

Flow rate $(\mathrm{ml} / \mathrm{min})$

Column temperature

Detector

Ionization source

Drying gas temperature

Drying gas flow rate

Nebulizer gas pressure
Perkin Elmer FX-10 Ultra High Performance Liquid Chromatography/SQ 300

Phenomenex Luna $5 \mu \mathrm{m} \mathrm{C18} \mathrm{(250x4.60} \mathrm{mm,} \mathrm{ID} 5 \mu \mathrm{m})$

Eluent A, water; eluent B, acetonitrile; gradient, 0-20 min (15-34.5\% B),

20-30 $\min (34.5-36 \% \mathrm{~B}), 30-40 \min (36-47.5 \% \mathrm{~B}), 40-45.2 \min (47.5-55 \% \mathrm{~B})$, 45.2-46.2 $\min (55-100 \% \mathrm{~B}), 46.2-60 \mathrm{~min}(100 \% \mathrm{~B})$

$1 \mathrm{ml} / \mathrm{min} \rightarrow 0.4 \mathrm{ml} / \mathrm{min}$ (split mode)

$20^{\circ} \mathrm{C}$

SQ 300 MS detector (single quadrupole)

ESI (electrospray ionization)

$300^{\circ} \mathrm{C}$

$10 \mathrm{l} / \mathrm{min}$

$80 \mathrm{psi}$

\section{A}

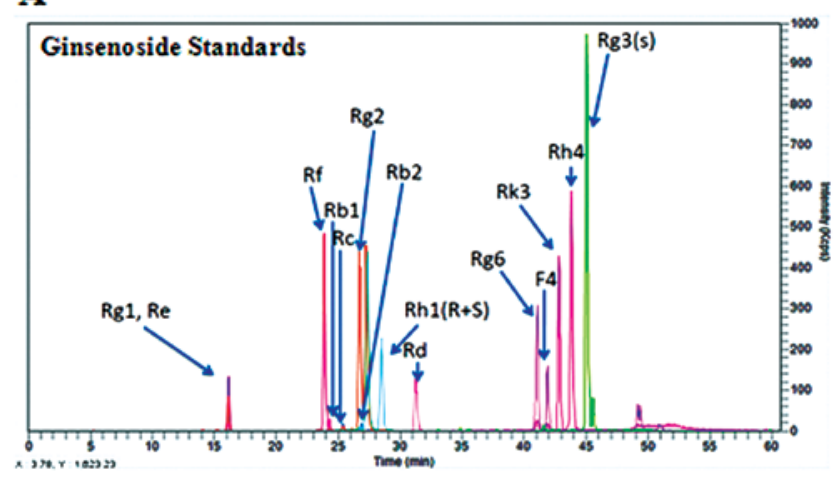

B

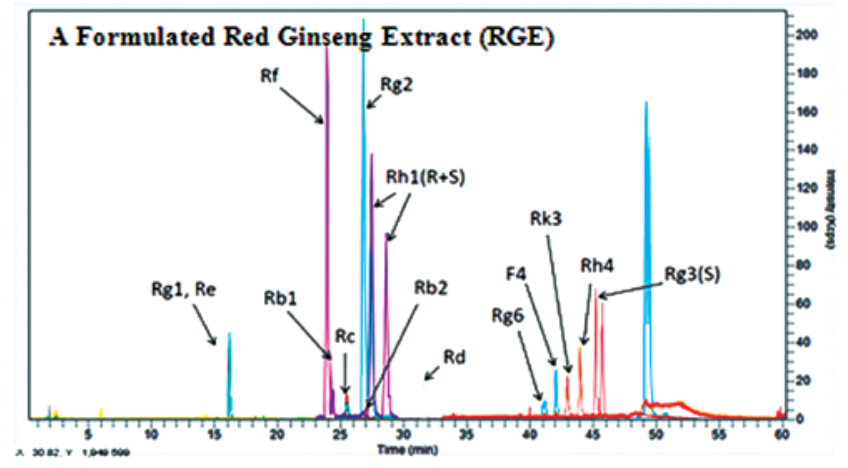

C

\begin{tabular}{|c|c|}
\hline Ginsenoside & $\underline{\mu} / \mathbf{m g}$ \\
\hline \hline $\mathrm{Rg} 1$ & 2.6 \\
$\mathrm{Re}$ & 2.13 \\
$\mathrm{Rb} 1$ & 5.26 \\
$\mathrm{Rc}$ & 4.99 \\
$\mathrm{Rb} 2$ & 2.01 \\
$\mathrm{Rd}$ & 0.86 \\
$\mathrm{Rg} 3(\mathrm{~S})$ & 0.26 \\
$\mathrm{Rf}$ & 2.3 \\
$\mathrm{Rg} 2$ & 1.91 \\
$\mathrm{Rh} 1(\mathrm{R} \div \mathrm{S})$ & 1.89 \\
$\mathrm{Rg} 6$ & 0.2 \\
$\mathrm{~F} 4$ & 0.81 \\
$\mathrm{Rk} 3$ & 0.18 \\
$\mathrm{Rh} 4$ & 0.22 \\
\hline
\end{tabular}

death, as evidenced by the EthD-1-positive cell line. We found that TRAIL ( $25 \mathrm{ng} / \mathrm{ml})$ induced poly (ADP-ribose) polymerase (PARP) cleavage at $12 \mathrm{~h}$, but that the RGE alone had little effect on PARP and caspase-3 cleavage (Fig. 2B).

TRAIL-derived cell death was augmented in the HCC cell lines by pretreatment with the RGE. Because our data suggested that the RGE alone has no obvious effect on cancer cell cytotoxicity, we then measured the sensitization effect of the RGE on TRAIL-derived cell death. When the RGE and TRAIL were added to cells consecutively, dramatic cell death occurred according to the results of the MTT colorimetric assay (Fig. 2C). Concentrations of TRAIL $>50 \mathrm{ng} / \mathrm{ml}$ were required to induce significant cytotoxicity; however, concentrations of TRAIL $<25 \mathrm{ng} / \mathrm{ml}$ showed minimal cytotoxicity. Viability measurements from concentration curves obtained from 1:2 serial dilutions demonstrated that $10 \mathrm{mg} / \mathrm{ml}$ of the RGE was necessary to achieve robust TRAIL-derived cell death (data not shown), but RGE alone showed a minimal cytotoxic effect. We further tested the sensitization effect in the Hep3B and Huh-7 cell lines. A similar degree of TRAIL $(25 \mathrm{ng} / \mathrm{ml})$ sensitization was observed, indicating that this effect was universal in HCC cell lines (Fig. 3A). In contrast, a combination of the RGE and TRAIL sensitize normal human liver cell lines, confirming that this sensitization was specific to the tumor cell lines (Fig. 3B).

One of the primary problems when clinically applying TRAIL as a cancer therapeutic agent is resistance. One effective strategy to surmount this issue would be to discover anticancer agents for use in combination therapy (26-29). Several natural products and other chemical compounds have been identified that sensitize cancer cell lines to TRAIL-derived apoptosis. In this study, we tested a formulated RGE that has been suggested to possess anticancer properties and the results provide substantial evidence that the RGE was capable of sensitizing

Figure 1. Analysis of the formulated RGE. (A) The liquid chromatography mass spectroscopy chromatogram (LC/MS) of the ginsenoside standards (reference compounds). (B) The LC/MS chromatogram of the formulated RGE. (C) Ginsenoside contents in the RGE. 
A
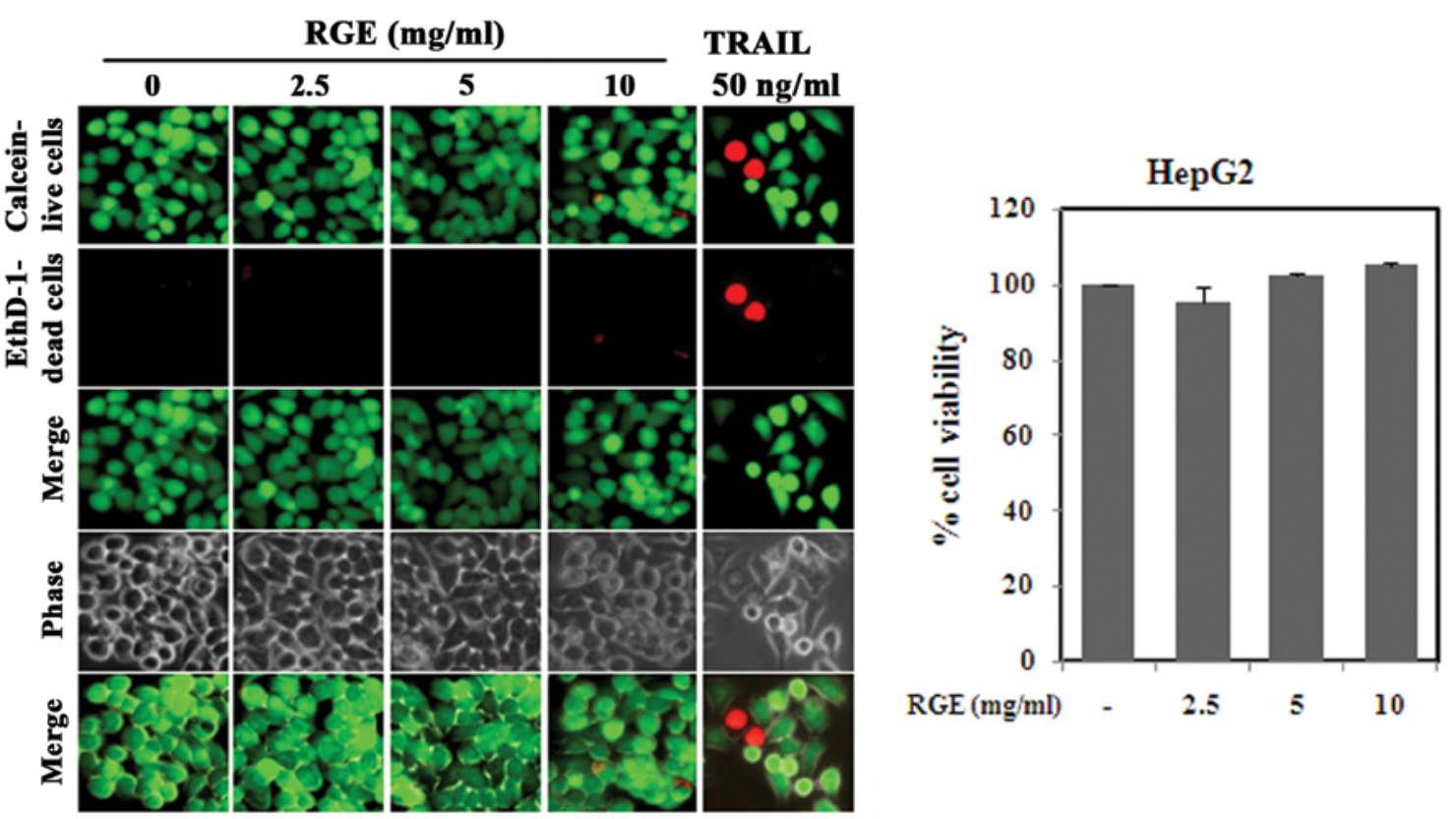

B

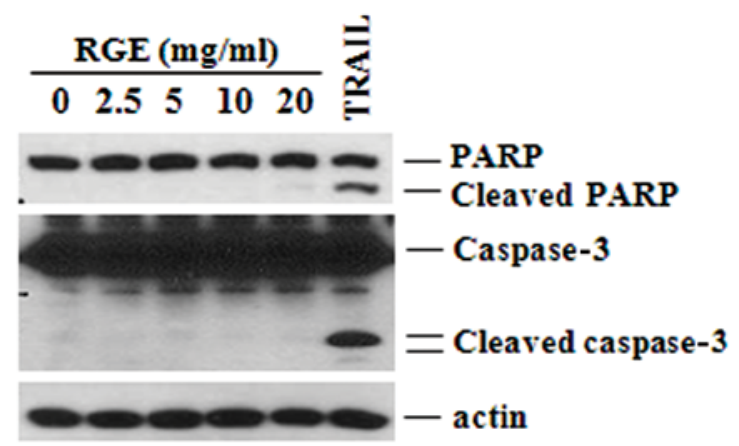

C
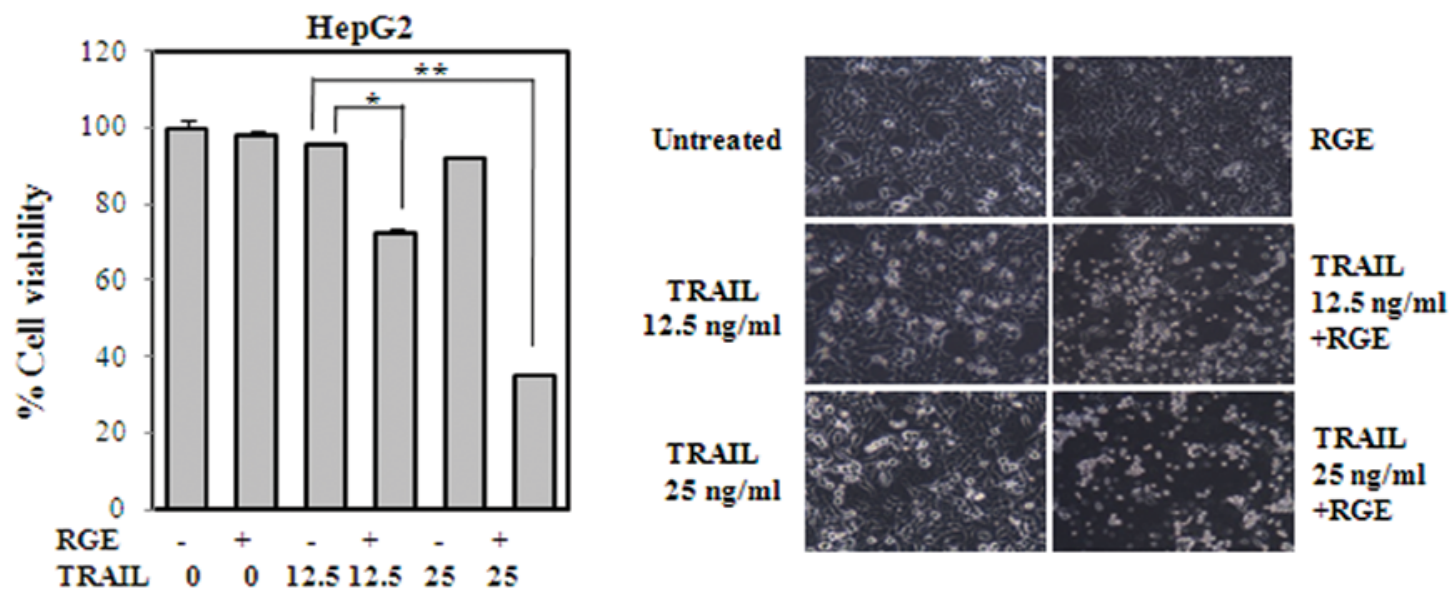

Figure 2. The RGE sensitizes HCC cell lines to TRAIL-derived cell death. (A) The RGE alone showed minimal anticancer effects in the HepG2 cells. Morphological signs of cell death and viability were analyzed by a cell death assay (left panel) and an MTT assay (right panel), respectively. (B) Cells were treated with the RGE (30 min) and TRAIL (12 h), sequentially. (C) Viable cells were measured using the MTT assay (left panel). Representative images were taken using a phasecontrast microscope (right panel). Results are expressed as mean \pm standard error. ${ }^{*} \mathrm{p}<0.001,{ }^{* *} \mathrm{p}<0.002$.

HCC to TRAIL-derived apoptosis by upregulating the CHOP, which then induced DR5 expression.

Sensitization effect of the RGE in TRAIL-derived cell death is caspase-dependent. We next examined the effects of the RGE on TRAIL activation of the caspase cascade in the SK-Hepl cell line. As shown in Fig. 3C, treatment with a combination of RGE and TRAIL induced cleavage of caspase-3 and PARP, a well-established caspase substrate, whereas treatment with the RGE alone did not. In addition, cell death was apoptotic because necrostatin-1 failed to inhibit cell death, as shown in Fig. 3D. To further confirm RGE and TRAIL-derived apoptosis, cells were 
A

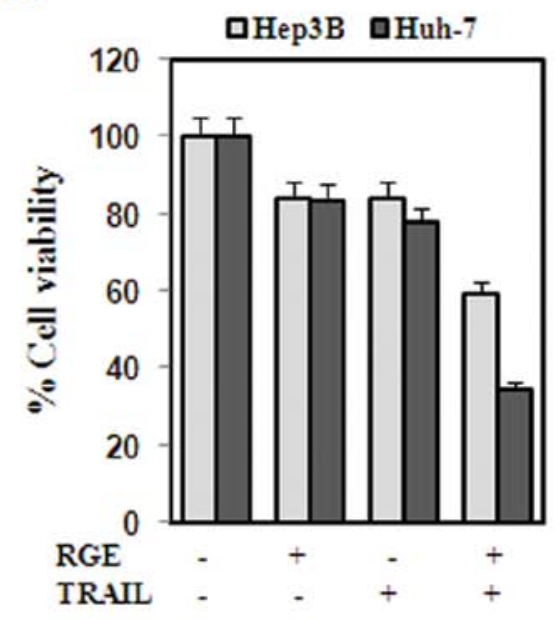

B

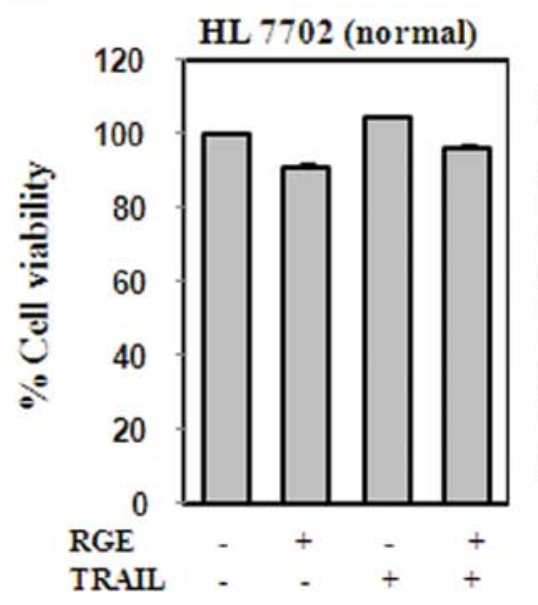

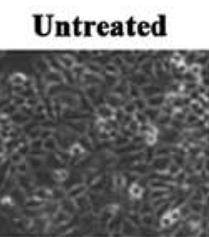
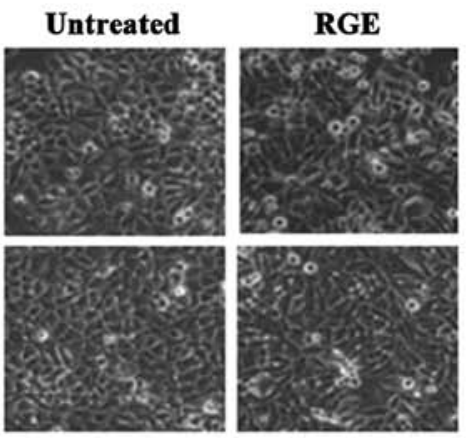

TRAIL

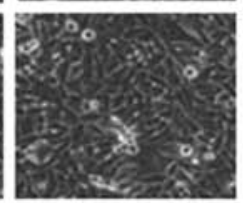

RGE+TRAIL

$\mathrm{C}$

D

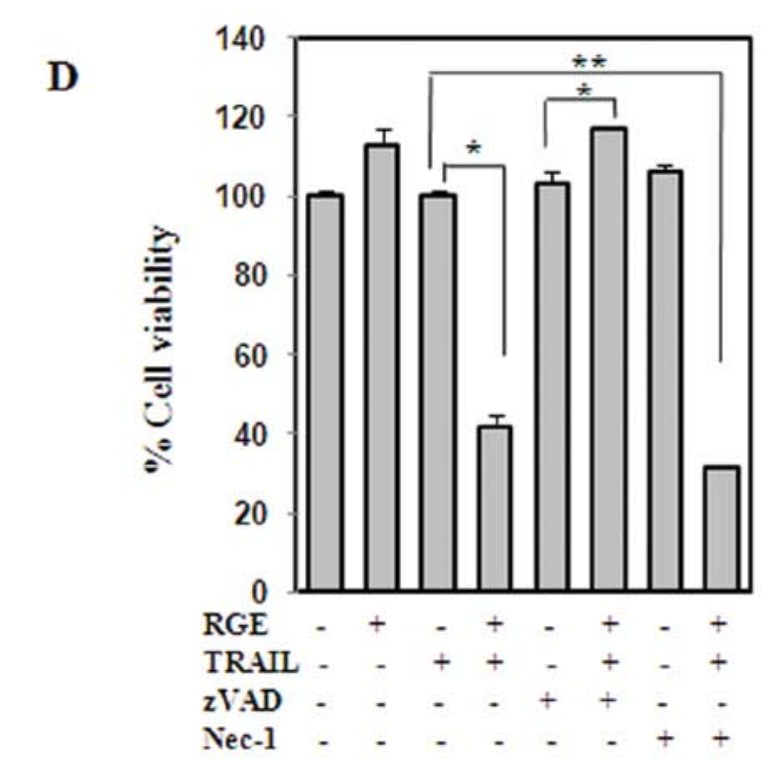

$\mathbf{E}$

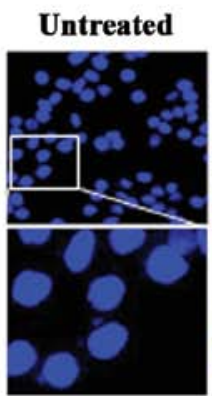

PARP

Cleaved

PARP

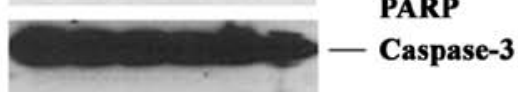

Cleaved

Caspase-3

- $\beta$-actin

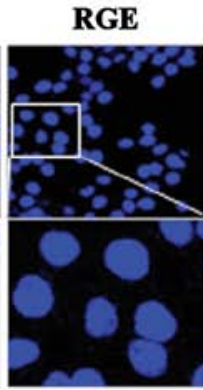

TRAIL RGE+TRAIL

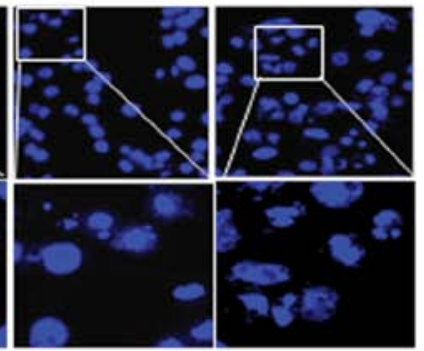

Figure 3. The RGE enhances TRAIL-derived apoptosis in HCC cell lines. (A) RGE sensitized TRAIL-derived cell death in different HCC cell lines. (B) RGE plus TRAIL-derived cell death was specific to the tumor cell lines. Cell viability was assessed using the MTT assay (left panel), and representative cell images were taken using a phase-contrast microscope (right panel). (C) The RGE enhanced activation of the caspase cascade triggered by TRAIL. RGE enhanced TRAIL-derived apoptosis. (D) Treatment with a caspase inhibitor blocked cell death in HepG2 cells. (E) Nuclear DNA fragmentation. Results are expressed as mean \pm standard error. ${ }^{*} \mathrm{p}<0.001,{ }^{* *} \mathrm{p}<0.002$.

cultured on coverslips and treated with RGE, TRAIL or RGE plus TRAIL. The cells were fixed and stained with DAPI after 16-18 $\mathrm{h}$, and examined for morphologic changes such as nuclear DNA fragmentation and/or condensation, which are indicative of apoptotic death. As shown in Fig. 3E, the RGE alone had no clear effect on nuclear DNA fragmentation and/or condensation, but RGE pretreatment significantly augmented TRAIL-derived nuclear DNA fragmentation and/or condensation in HepG2 cells.

$R G E$ induces upregulation of DR5 expression. TRAIL resistance is facilitated by the downregulation of DR4 and 
A

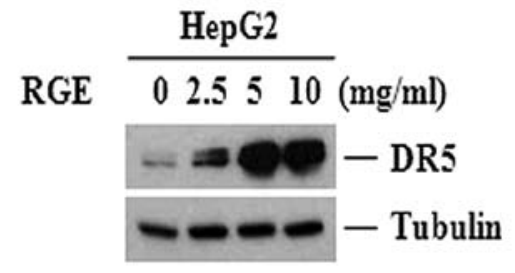

B

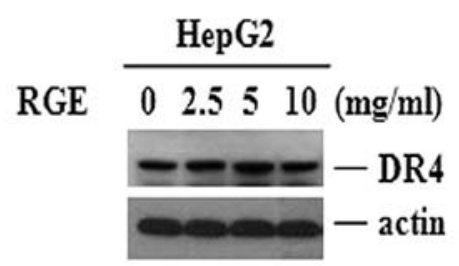

C

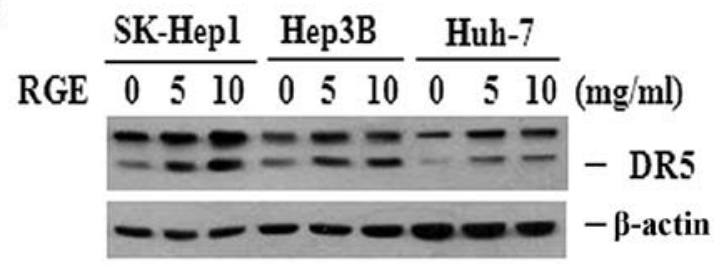

DR5 and upregulation of DcR1 and DcR2 (8,30-32). We examined TRAIL receptor protein levels in cells treated with the RGE to explore the molecular mechanisms underlying the sensitization effect of the RGE to TRAIL-derived cell death. Treatment with the RGE significantly increased the expression of DR5 in a dose-dependent manner in HepG2 cells (Fig. 4A), although no clear changes were found in DR4 expression (Fig. 4B). We compared the effects of the RGE in three different HCC cell lines to determine whether this effect was cell-type specific (Fig. 4C). All cell lines tested showed upregulated DR5 expression following the RGE treatment, suggesting that the effects of RGE on the upregulation of DR5 are not cell-type specific.

Figure 4. The RGE sensitization to TRAIL-derived cell death occurs via death receptor 5 (DR5) upregulation in HCC cell lines. (A) DR5 protein levels were strongly dependent upon RGE concentration. (B) RGE-induced upregulation of DR5, but not DR4, in HepG2 cells. (C) Upregulation of DR5 protein levels by RGE treatment in the different $\mathrm{HCC}$ cell lines.
A

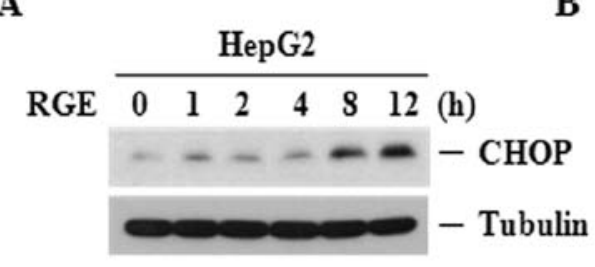

B

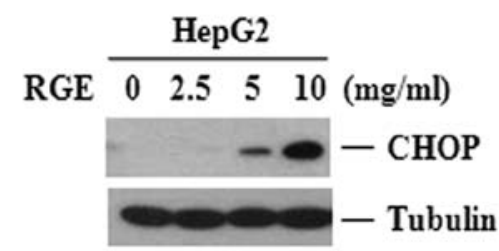

$\mathrm{C}$

$$
\begin{aligned}
& \text { RGE } \begin{array}{llllllllllll} 
& 0 & 5 & 10 & 0 & 5 & 10 & 0 & 5 & 10 & (\mathrm{mg} / \mathrm{ml})
\end{array} \\
& \begin{array}{l}
-\infty-\infty-\infty-\text { CHOP } \\
-\infty-\infty-\text { Tubulin }
\end{array}
\end{aligned}
$$

D

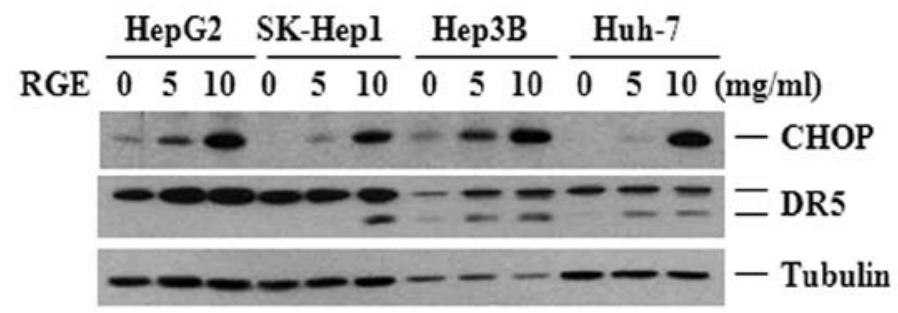

E

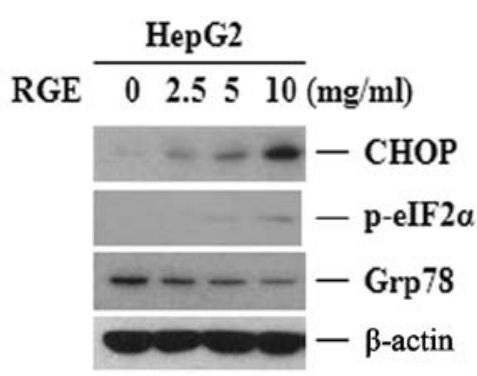

$\mathbf{F}$

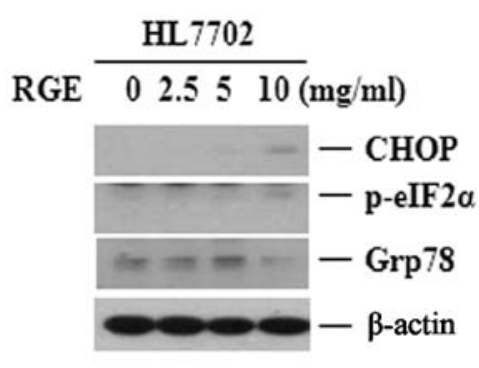

Figure 5. RGE-induced DR5 upregulation is mediated by induction of the CHOP in HCC cell lines. (A and B) Upregulation of CHOP levels was strongly dependent upon (A) treatment time and (B) RGE concentration. (C and D) Immunoblotting of HCC cell lysates treated with different concentrations of the RGE for $12 \mathrm{~h}$. (E and F) Western blot analysis of (E) HepG2 and (F) HL 7702 cell lysates. 


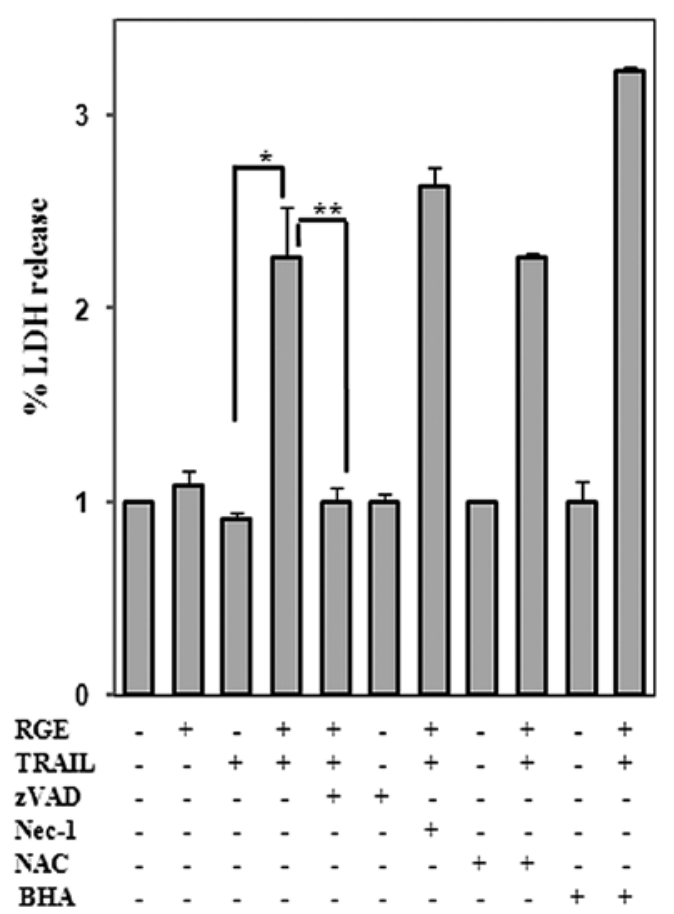

Figure 6. ROS are not required for RGE sensitization to TRAIL-derived cell death. Cell cytotoxicity was determined using the LDH assay. The results are shown as averages \pm SEM. ${ }^{*} \mathrm{p}<0.01,{ }^{* * *} \mathrm{p}<0.02$.

Many mechanisms have been elucidated for inducing DR5, including ER stress, p53 induction, reactive oxygen species (ROS) generation and NF- $\mathrm{KB} / \mathrm{MAPK}$ activation (33-35). It has been suggested that C/EBP homologous protein/GADD153, a transcription factor of the C/EBP family that has a role in ER stress, is involved in activating DR5 expression and thus contributes to the effectiveness of the RGE and TRAIL combined therapy (36-38). The RGE treatment increased CHOP levels in the HepG2 cell lines corresponding to its sensitization effect on TRAIL-derived cell death (Fig. 5A-C). As shown in Fig. 5D, RGE-induced CHOP upregulation preceded the increase in DR5 levels. Such a temporal pattern further supports the notion that CHOP plays a role in the activation of DR5 expression by the RGE. As CHOP is a major transcription factor induced by ER stress, we examined whether RGE treatment would induce ER stress in HCC. Thus, we probed for two markers of ER stress: Ser-51 phosphorylation of eIF2 $\alpha$ (P-eIF2 $\alpha$ ) and induction of the ER chaperone protein GRP78. As shown in Fig. 5E, the RGE upregulated C/EBP homologous protein but did not induce GRP78 or eIF2 $\alpha$ phosphorylation in the HepG2 cell lines, indicating that the RGE was capable of over-expressed the CHOP without substantively affecting ER stress. However, the RGE did not influence CHOP expression in the normal liver cell lines, which strongly agrees with the observation that the RGE did not boost the effect of TRAIL in the normal cell lines (Fig. 3B). Additionally, the RGE was unable to induce expression of the $\mathrm{C} / \mathrm{EBP}$ homologous protein or other ER stress markers in the normal cell lines (Fig. 5F).

When investigating the induction of the TRAIL receptors DR4 and DR5 in tumors it is very important to assess susceptibility of the tumor to TRAIL treatment (39). Several studies have shown that high expression of the TRAIL receptors DR4
A

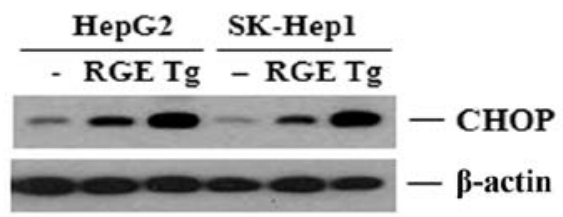

B

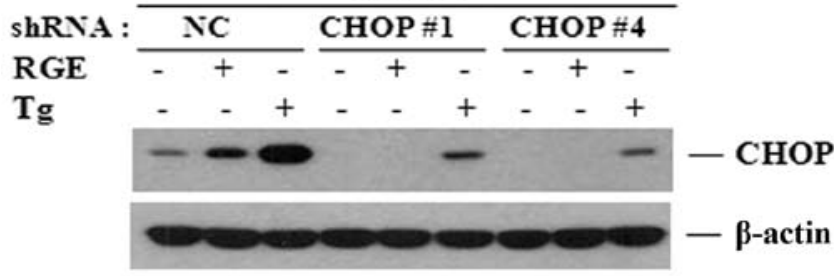

C

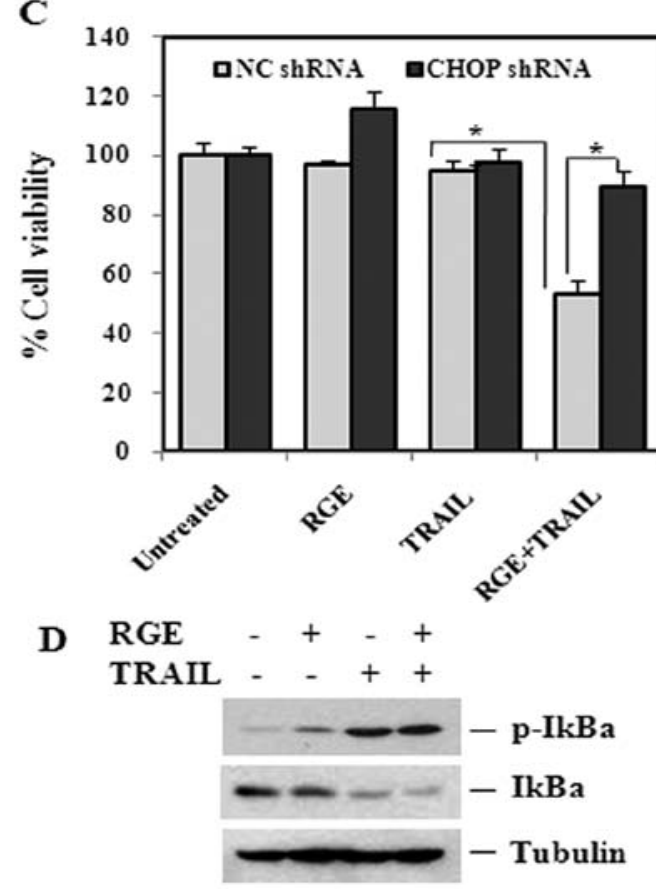

Figure 7. shRNA inhibits CHOP protein expression and therefore hinders the effect of the RGE and TRAIL combined treatment. (A) RGE and Tg induced upregulation of CHOP expression in the HepG2 and SK-Hep1 cell lines. (B) CHOP expression was effectively inhibited by shRNA. (C) Downregulation of CHOP suppressed RGE-triggered enhancement of TRAIL-derived apoptosis in lentivirus-infected HepG2 cells. (D) SK-Hep1 cells were treated with the RGE and a combination of the RGE and TRAIL for $16 \mathrm{~h}$. Results are expressed as mean \pm standard error. ${ }^{*} \mathrm{p}<0.02,{ }^{* *} \mathrm{p}<0.05$.

and DR5 facilitates sensitization of cancer cells to TRAILderived cell death $(1,40)$. One therapeutic approach being tested is to induce the expression of death receptors, including DR4 and particularly DR5, by small molecules, which results in TRAIL-derived tumor cell death or sensitization of TRAILresistant cell lines to cell death (33). Furthermore, DR5 expression levels are highly correlated with TRAIL, which binds to its receptors, DR4 and DR5, and activates the extrinsic apoptosis pathway $(34,41,42)$. In the present study, we found that the improved effects of the combination therapy were primarily dependent upon control of DR5 induction. We also found that the combination therapy induced apoptosis in the 
presence of ROS (Fig. 6), suggesting that ROS do not contribute to RGE-facilitated sensitization of TRAIL-derived cell death.

Increased expression of CHOP is related to DR5 upregulation. $\mathrm{Tg}$ treatment was compared with RGE treatment to confirm that upregulation of CHOP plays a key role in the RGE sensitization effect, as shown in Fig. 7A (43,44). Next, we established stable cell lines for knockdown of the C/EBP homologous protein using a lentiviral shRNA system. As shown in Fig. 7B, we confirmed knockdown of CHOP. In addition, knockdown of CHOP inhibited the robust upregulation of CHOP induced by $\mathrm{Tg}$. We then examined the effect of CHOP knockdown on RGE-induced sensitization to TRAIL-derived cell death. CHOP knockdown inhibited the effect of the RGE plus TRAIL treatment in HepG2 cell lines, suggesting that augmentation of cell death by the RGE combined treatment in HCC was mediated by CHOP expression (Fig. 7C).

Activation of NF-KB in cancer cell lines contributes to the induction of DR5 as well as resistance to TRAIL-derived apoptosis. Therefore, we examined whether the RGE might regulate $\mathrm{NF}-\kappa \mathrm{B}$ to facilitate DR5 upregulation and, consequently, sensitize cell lines to TRAIL-derived apoptosis. As shown in Fig. 7D, degradation and phosphorylation of IкB $\alpha$ did not occur in response to the RGE. In addition, the RGE did not affect TRAIL-derived NF- $\mathrm{kB}$ activation, suggesting that RGE-induced sensitization to cell death is not mediated by NF- $\mathrm{KB}$. Although TRAIL is capable of activating NF- $\kappa B$ in some cancer cell lines and the induction of DR5 is linked to NF- $\mathrm{KB}$ activation, activated NF- $\mathrm{KB}$ also upregulates the anti-apoptotic gene Bcl-xL, effectively blocking TRAIL-derived apoptosis $(10,27,34)$. We failed to detect upregulation of $\mathrm{Bcl}-\mathrm{xL}$ in response to RGE treatment (data not shown), which further indicates that NF- $\mathrm{\kappa B}$ was activated by the RGE. Additionally, RGE-induced CHOP upregulation was not caused by ER stress, which can lead to apoptosis in HCC cell lines. Inducing CHOP expression or TRAIL sensitization was unsuccessful when RGE was applied to the normal cell line HL7702, suggesting that CHOP protein upregulation by the RGE is specific to the tumor cell lines.

Although we have not tested the antitumor activity of the RGE in vivo, the formulated RGE examined could potentially be further developed as an important chemosensitizer to be utilized as a dietary supplement with anticancer drugs.

\section{Acknowledgements}

This study was supported by the 2011 grant from the Korean Society of Ginseng funded by Korea Ginseng Corporation and the Yujeonja-Donguibogam project based on Traditional herbs (Grant No. 2012M3A9C4048796), Republic of Korea. This study was also supported by a National Research Foundation of Korea (NRF) grant funded by the Korean government (20110030834) to Y.-S. Kim and by the 2010 grant from Department of Medical Sciences, the Graduate School, Ajou University.

\section{References}

1. Kischkel FC, Lawrence DA, Chuntharapai A, Schow P, Kim KJ and Ashkenazi A: Apo2L/TRAIL-dependent recruitment of endogenous FADD and caspase- 8 to death receptors 4 and 5 . Immunity 12: 611-620, 2000.
2. Deveraux QL, Roy N, Stennicke HR, et al: IAPs block apoptotic events induced by caspase- 8 and cytochrome $\mathrm{c}$ by direct inhibition of distinct caspases. EMBO J 17: 2215-2223, 1998.

3. $\mathrm{Hu}$ W, Johnson $\mathrm{H}$ and Shu H: Tumor necrosis factor-related apoptosis-inducing ligand receptors signal NF- $\kappa \mathrm{B}$ and JNK activation and apoptosis through distinct pathways. J Biol Chem 274: 30603, 1999.

4. Lin B, Williams-Skipp C, Tao Y, et al: NF-кB functions as both a proapoptotic and antiapoptotic regulatory factor within a single cell type. Cell Death Differ 6: 570-582, 1999.

5. Son YG, Kim EH, Kim JY, et al: Silibinin sensitizes human glioma cells to TRAIL-mediated apoptosis via DR5 up-regulation and down-regulation of c-FLIP and survivin. Cancer Res 67: 8274-8284, 2007.

6. Ding L, Yuan C, Wei F, et al: Cisplatin restores TRAIL apoptotic pathway in glioblastoma-derived stem cells through up-regulation of DR5 and down-regulation of c-FLIP. Cancer Invest 29: 511-520, 2011.

7. Park SJ, Bijangi-Vishehsaraei $K$ and Safa AR: Selective TRAIL-triggered apoptosis due to overexpression of TRAIL death receptor 5 (DR5) in P-glycoprotein-bearing multidrug resistant CEM/VBL1000 human leukemia cells. Int J Biochem Mol Biol 1: 90-100, 2010

8. Fossati S, Ghiso J and Rostagno A: TRAIL death receptors DR4 and DR5 mediate cerebral microvascular endothelial cell apoptosis induced by oligomeric Alzheimer's A $\beta$. Cell Death Dis 3: e321, 2012.

9. Griffith TS, Fialkov JM, Scott DL, et al: Induction and regulation of tumor necrosis factor-related apoptosis-inducing ligand/ Apo-2 ligand-mediated apoptosis in renal cell carcinoma. Cancer Res 62: 3093-3099, 2002.

10. Kim JY, Lee JY, Kim DG, Koo GB, Yu JW and Kim YS: TRADD is critical for resistance to TRAIL-induced cell death through NF-kB activation. FEBS Lett 585: 2144-2150, 2011.

11. Tian X, Ye J, Alonso-Basanta M, Hahn SM, Koumenis C and Dorsey JF: Modulation of CCAAT/enhancer binding protein homologous protein (CHOP)-dependent DR5 expression by nelfinavir sensitizes glioblastoma multiforme cells to tumor necrosis factor-related apoptosis-inducing ligand (TRAIL). J Biol Chem 286: 29408-29416, 2011.

12. Carr BI: Hepatocellular carcinoma: current management and future trends. Gastroenterology 127: S218-S224, 2004.

13. Yang JF, Cao JG, Tian L and Liu F: 5,7-Dimethoxyflavone sensitizes TRAIL-induced apoptosis through DR5 upregulation in hepatocellular carcinoma cells. Cancer Chemother Pharmacol 69: 195-206, 2012.

14. Zhang B, Shan H, Li D, et al: Cisplatin sensitizes human hepatocellular carcinoma cells, but not hepatocytes and mesenchymal stem cells, to TRAIL within a therapeutic window partially depending on the upregulation of DR5. Oncol Rep 25: 461-468, 2011.

15. Lee SA, Jo HK, Im BO, Kim SU, Whang WK and Ko SK: Changes in the Contents of Prosapogenin in the Red Ginseng (Panax ginseng) Depending on Steaming Batches. J Ginseng Res 36: 102-106, 2012.

16. In G, Ahn NG, Bae BS, Han ST, Noh KB and Kim CS: New method for simultaneous quantification of 12 ginsenosides in red ginseng powder and extract: in-house method validation. J Ginseng Res 36: 205-210, 2012.

17. Sun S, Qi LW, Du GJ, Mehendale SR, Wang CZ and Yuan CS: Red notoginseng: higher ginsenoside content and stronger anticancer potential than Asian and American ginseng. Food Chem 125: 1299-1305, 2011.

18. Keum YS, Park KK, Lee JM, et al: Antioxidant and anti-tumor promoting activities of the methanol extract of heat-processed ginseng. Cancer Lett 150: 41-48, 2000.

19. Li J, Huang M, Teoh $\mathrm{H}$ and Man RY: Panax quinquefolium saponins protects low density lipoproteins from oxidation. Life Sci 64: 53-62, 1999.

20. Chang MS, Lee SG and Rho HM: Transcriptional activation of $\mathrm{Cu} / \mathrm{Zn}$ superoxide dismutase and catalase genes by panaxadiol ginsenosides extracted from Panax ginseng. Phytother Res 13: 641-644, 1999.

21. Ji HY, Lee HW, Kim HK, et al: Simultaneous determination of ginsenoside $\mathrm{Rb}(1)$ and $\mathrm{Rg}(1)$ in human plasma by liquid chromatography-mass spectrometry. J Pharm Biomed Anal 35: 207-212, 2004. 
22. Cai F, Sun L, Gao S, Yang Y, Yang Q and Chen W: A rapid and sensitive liquid chromatography-tandem mass spectrometric method for the determination of timosaponin B-II in blood plasma and a study of the pharmacokinetics of saponin in the rat. J Pharm Biomed Anal 48: 1411-1416, 2008.

23. Woo SS, Song JS, Lee JY, et al: Selection of high ginsenoside producing ginseng hairy root lines using targeted metabolic analysis. Phytochemistry 65: 2751-2761, 2004.

24. Park JD, Rhee DK and Lee YH: Biological activities and chemistry of saponins from Panax ginseng CA Meyer. Phytochem Rev 4: 159-175, 2005.

25. Lee DCW and Lau ASY: Effects of Panax ginseng on tumor necrosis factor- $\alpha$-mediated inflammation: a mini-review. Molecules 16: 2802-2816, 2011.

26. Sayers TJ: Targeting the extrinsic apoptosis signaling pathway for cancer therapy. Cancer Immunology, Immunotherapy: pp1-8, 2011.

27. Ravi R, Bedi GC, Engstrom LW, et al: Regulation of death receptor expression and TRAIL/Apo2L-induced apoptosis by NF-kB. Nat Cell Biol 3: 409-416, 2001.

28. Ganten TM, Koschny R, Sykora J, et al: Preclinical differentiation between apparently safe and potentially hepatotoxic applications of TRAIL either alone or in combination with chemotherapeutic drugs. Clin Cancer Res 12: 2640-2646, 2006.

29. Baritaki S, Huerta-Yepez S, Sakai T, Spandidos DA and Bonavida B: Chemotherapeutic drugs sensitize cancer cells to TRAIL-mediated apoptosis: up-regulation of DR5 and inhibition of Yin Yang 1. Mol Cancer Ther 6: 1387-1399, 2007.

30. Mellier G, Huang S, Shenoy K and Pervaiz S: TRAILing death in cancer. Mol Aspects Med 31: 93-112, 2010

31. Kouhara J, Yoshida T, Nakata S, et al: Fenretinide upregulates DR5/TRAIL-R2 expression via the induction of the transcription factor CHOP and combined treatment with fenretinide and TRAIL induces synergistic apoptosis in colon cancer cell lines. Int J Oncol 30: 679-687, 2007.

32. Bertram H, Nerlich A, Omlor G, Geiger F, Zimmermann G and Fellenberg J: Expression of TRAIL and the death receptors DR4 and DR5 correlates with progression of degeneration in human intervertebral disks. Mod Pathol 22: 895-905, 2009.

33. Xiaowen $\mathrm{H}$ and $\mathrm{Yi}$ S: Triptolide sensitizes TRAIL-induced apoptosis in prostate cancer cells via p53-mediated DR5 up-regulation. Mol Biol Rep 39: 8763-8770, 2012.
34. Lin FL, Hsu JL, Chou CH, Wu WJ, Chang CI and Liu HJ: Activation of p38 MAPK by damnacanthal mediates apoptosis in SKHep 1 cells through the DR5/TRAIL and TNFR1/TNF- $\alpha$ and p53 pathways. Eur J Pharmacol 650: 120-129, 2011.

35. Deng Z, Yan H, Hu J, et al: Hepatitis $\mathrm{C}$ virus sensitizes host cells to TRAIL-induced apoptosis by up-regulating DR4 and DR5 via a MEK1-dependent pathway. PloS One 7: e37700, 2012.

36. Shiraishi T, Yoshida T, Nakata S, et al: Tunicamycin enhances tumor necrosis factor-related apoptosis-inducing ligandinduced apoptosis in human prostate cancer cells. Cancer Res 65: 6364-6370, 2005.

37. Chen CY, Yiin SJ, Hsu JL, Wang WC, Lin SC and Chern CL: Isoobtusilactone A sensitizes human hepatoma Hep G2 cells to TRAIL-induced apoptosis via ROS and CHOP-mediated up-regulation of DR5. J Agric Food Chem 60: 3533-3539, 2012.

38. Oyadomari S and Mori M: Roles of CHOP/GADD153 in endoplasmic reticulum stress. Cell Death Differ 11: 381-389, 2003.

39. Bin L, Thorburn J, Thomas LR, Clark PE, Humphreys R and Thorburn A: Tumor-derived mutations in the TRAIL receptor DR5 inhibit TRAIL signaling through the DR4 receptor by competing for ligand binding. J Biol Chem 282: 28189-28194, 2007.

40. Sheridan JP, Marsters SA, Pitti RM, et al: Control of TRAIL-induced apoptosis by a family of signaling and decoy receptors. Science 277: 818-821, 1997.

41. Seol DW: p53-Independent up-regulation of a TRAIL receptor DR5 by proteasome inhibitors: a mechanism for proteasome inhibitor-enhanced TRAIL-induced apoptosis. Biochem Biophys Res Commun 416: 222-225, 2011.

42. Sung B, Ravindran J, Prasad S, Pandey MK and Aggarwal BB: Gossypol induces death receptor-5 through activation of the ROS-ERK-CHOP pathway and sensitizes colon cancer cells to TRAIL. J Biol Chem 285: 35418-35427, 2010.

43. Zinszner H, Kuroda M, Wang X, et al: CHOP is implicated in programmed cell death in response to impaired function of the endoplasmic reticulum. Genes Dev 12: 982-995, 1998

44. Zou W, Yue P, Khuri F and Sun S: Coupling of endoplasmic reticulum stress to CDDO-Me-induced up-regulation of death receptor 5 via a CHOP-dependent mechanism involving JNK activation. Cancer Res 68: 7484-7492, 2008. 